$\circlearrowleft$

\title{
Propofol: avaliação clínica e laboratorial em cães
}

\section{Propofol: clinical and laboratorial evaluation in dogs}

\author{
Rosana Pinheiro Botelho ${ }^{1}$, Marcilio Dias do Nascimento ${ }^{2}$, Firmino Marsico Filho ${ }^{2}$
}

\begin{abstract}
Resumo
Foram utilizados 7 cães (Canis familiaris) para indução e manutenção em bolus anestésico de propofol, sem préanestesia, durante um período de $60 \mathrm{~min}$. Foram avaliados os parâmetros clínicos: freqüência cardíaca, freqüência respiratória, reflexos palpebral e interdigital; laboratoriais: hematológicos - hematimetria, volume globular, hemoglobinometria, volume globular médio, concentração de hemoglobina globular média, leucometrias global e específica; bioquímicos - aspartato amino transferase, alanina amino transferase, uréia, creatinina, glicose, proteínas plasmáticas totais, albumina, globulinas e relação albumina/globulinas; e urinários - nitrito, $\mathrm{pH}$, proteína, glicose, cetona, urobilinogênio, bilirrubina e sangue. $O$ propofol determinou apnéia, leucopenia, desvio à esquerda sem neutrofilia, aumento dos valores da uréia e diminuição de proteínas plasmáticas totais, globulinas e albumina; causou alteração significativa nos valores da leucometria global, nos bastões e na freqüência do pulso.
\end{abstract}

Palavras chave: propofol; anestesia; hematologia; bioquímica; urinálise; cão

\section{Introdução}

Várias drogas do arsenal terapêutico anestesiológico da medicina humana são empregadas rotineiramente em veterinária.

A utilização de drogas mais efetivas e menos tóxicas e o aperfeiçoamento das técnicas de administração das mesmas têm sido objeto de uma intensa procura a fim de melhorar a qualidade da anestesia. Uma técnica que atraiu a atenção de muitos anestesiologistas nos últimos 10 anos foi a anestesia venosa total:

O propofol, um anestésico venoso do grupo dos alquifenóis, foi incorporado ao arsenal anestesiológico como agente indutor e de manutenção, através de bolus e/ou em infusão contínua. Apresenta-se sob a forma de uma emulsão de óleo em água, contendo $200 \mathrm{mg}$ em $20 \mathrm{ml}$ (1\%), que tem cor branca, $\mathrm{pH}$ neutro, é estável, isotônica, estéril e pronta para uso; seu armazenamento deve ser feito a temperatura ambiente, inferior a $25^{\circ} \mathrm{C}$, não devendo ser congelada e necessita ser agitada antes do uso; pos- sui efeitos cumulativos mínimos, além de não determinar respostas anafilactóides ou liberação de histamina (Wellcome, 1989).

Vários são os trabalhos sobre anestesia venosa no cão (Canis familiaris) utilizando o propofol através da indução com bolus, sem pré-anestesia (Watkins et al., 1987; Genevois et al., 1988; Searle, 1989; Morgan e Legge, 1989; Jones, 1990; Quandt e Robinson, 1991, 1992; Mandsager et al., 1991; Robertson et al., 1991; Marsico et al., 1991).

Nos trabalhos de Genevois et al. (1988), Marsico et al. (1991) e Mandsager et al. (1991), foi adotada a técnica de indução com bolus de propofol sem manutenção anestésica. Watkins et al. (1987), Morgan e Legge (1989) e Jones (1990) instituíram as técnicas de indução e manutenção com bolus de propofol, enquanto que Searle (1989) e Quandt e Robinson (1991) utilizaram técnicas de indução com bolus e manutenção em infusão contínua.

Parâmetros clínicos, tais como freqüência cardíaca (FC) e freqüência respiratória (FR), foram avaliados por Quandt e Robinson (1992), que não encontraram alterações cardiovasculares significativas após o uso do propofol. Marsico et al. (1991) observaram que a FC aumentou e a FR diminuiu após a indução, enquanto que Quandt e Robinson (1991) concluíram que a depressão respiratória é comum após a indução. Mandsager et al. (1991) observaram bradicardia sinusal. Watkins et al. (1987), verificaram que os animais tiveram alterações nesses parâmetros por provável inquietação.

Segundo Langley e Heel (1988), a eliminação do propofol no homem é bifásica, sendo a primeira fase rápida, com meia vida de 25 a 56 min., enquanto que a fase terminal é de 184 a 309 min., após uma dose única dessa droga, e de 277 a 403 min. em seguida à infusão contínua.

A dose de indução com bolus de propofol sem préanestesia, adotada por vários autores (Watkins et al., 1987; Genevois et al., 1988; Morgan e Legge, 1989; Searle, 1989; Jones, 1990; Mandsager et al., 1991; Quandt e Robinson, 1991, 1992; Marsico et al., 1991), variou entre 5,95 e 9,40 $\mathrm{mg} / \mathrm{kg}$. A dose de manutenção com bolus de propofol, observada por Watkins et al. (1987) foi $0,806 \pm 0,413 \mathrm{mg} /$ $\mathrm{kg} / \mathrm{min}$., por Morgan e Legge (1989) $1,296 \mathrm{mg} / \mathrm{kg} / \mathrm{min}$. e por Jones (1990) 0,806 mg/kg/min. Searle (1989) e Quandt e Robinson (1991) utilizaram a técnica de manutenção em 
infusão contínua de propofol, adotando as doses, respectivamente, de $0,81 \mathrm{mg} / \mathrm{kg} / \mathrm{min}$. a $0,60 \mathrm{mg} / \mathrm{kg} / \mathrm{min}$. nos primeiros $30 \mathrm{~min}$. e de $0,80 \mathrm{mg} / \mathrm{kg} / \mathrm{min}$. dos 31 aos $60 \mathrm{~min}$.

Alguns autores (Watkins et al., 1987; Quandt e Robinson, 1991; Mandsager et al., 1991) observaram a ocorrência de apnéia após a indução, em 11 de 68 (16,2\%), em 2 de $5(40 \%)$ e em 5 de 10 animais (50\%), respectivamente. Esses autores, além de Genevois et al. (1988) Morgan e Legge (1989) e Marsico et al. (1991), observaram uma variação na duração da apnéia de 10 a 60 seg., podendo ocorrer por períodos de $3 \mathrm{~min}$.

No trabalho de Watkins et al. (1987) foram utilizados 104 cães; 44 não tiveram alterações de pulso, 35 tiveram aumento da freqüência e 25 diminuição da mesma, efeitos atribuídos à inquietação. Jones (1990) observou os mesmos efeitos cardiovasculares e respiratórios, enquanto Marsico et al. (1991) observaram que, além desses efeitos, o propofol produz depressão cardio-respiratória transitória dose-dependente.

Um estudo hematológico em cinco ensaios realizados pelo Laboratório Wellcome (1989) em 150 pacientes humanos submetidos a associações anestésicas em cirurgias diversas, inclui aferições de aspartato amino transferase (AST), alanina amino transferase (ALT), volume globular (VG), hemoglobulinometria $(\mathrm{Hb})$, contagem de leucócitos, uréia e creatinina. Cinco pacientes tiveram valores baixos de $\mathrm{Hb}$ ou VG no pós-operatório, mas como estavam anêmicos antes da cirurgia, os resultados não foram considerados como efeito do propofol; dois estavam com contagens de leucócitos inferiores aos limites normais após administração do propofol, embora já apresentassem valores baixos antes da anestesia. Os valores de uréia e creatinina se mantiveram dentro da faixa de normalidade. Concluiu-se que não existem evidências claras de que o propofol afete adversamente as funções hepáticas e renais ou os parâmetros hematológicos. Observou-se também que o propofol tem um elevado grau de ligação com proteínas plasmáticas humanas (97 a 98\%).

O objetivo deste trabalho foi a avaliação de alterações clínicas, hematológicas, bioquímicas e urinárias em cães submetidos à manutenção anestésica com propofol em bolus.

\section{Material e Métodos}

Foram utilizados 7 cães (Canis familiaris), sem raça determinada, 4 machos e 3 fêmeas, com pesos variando entre 7,7 e $29,1 \mathrm{~kg}$, mantidos em canis do Hospital Veterinário da Universidade Federal Rural do Rio de Janeiro, que receberam manejo padronizado, além de jejum e dieta hídrica por $15 \mathrm{~h}$, antes do início do experimento.
Os animais foram submetidos à anestesia com propofol a 1\% para avaliação de parâmetros clínicos e laboratoriais, bem como das doses anestésicas e da ocorrência e duração de apnéia.

A indução anestésica lenta, com bolus de propofol, foi realizada até a perda dos reflexos palpebral e interdigital, seguindo-se a manutenção com bolus durante $60 \mathrm{~min}$., administrado quando necessário. Durante a manutenção anestésica os animais foram mantidos com infusão contínua de cloreto de sódio a $0,9 \%$ na dosagem de $10 \mathrm{ml} / \mathrm{kg} / \mathrm{h}$.

Os dados laboratoriais foram coletados nos tempos I, II, III e IV, que corresponderam, respectivamente, aos tempos antes da indução anestésica, aos 60 e $300 \mathrm{~min}$. e às $24 \mathrm{~h}$ após a indução anestésica. Os dados clínicos foram coletados nos tempos 0 min. a 60 min. (t 0 a t 60), com intervalos de 5 ou de $10 \mathrm{~min}$. (Os números representados ao lado da letra t, de t 0 a t 60 , representam os minutos decorridos desde a indução anestésica).

Após tricotomia, assepsia e punção da veia jugular, procedeu-se à coleta de sangue para realização do hemograma e determinação das proteínas plasmáticas totais $(\mathrm{PPT})^{1} \mathrm{e}$ da albumina $(A L B)^{1}$, de soro para determinações de uréia, creatinina ${ }^{1}, A T^{1}$ e $A L T^{1}$ e de plasma fluoretado para a glicemia ${ }^{1}$. As globulinas (GLOB) foram determinadas pela diferença entre PPT e ALB, enquanto a relação $A L B / G L O B$ foi determinada dividindo-se o valor de ALB pelo valor das GLOB. A urina foi obtida através de cateterismo vesical para realização dos exames bioquímicos com fitas reagentes ${ }^{2}$.

A FC e a FR foram registradas a cada 10 min., desde 0 a 60 ; os reflexos palpebral e interdigital foram registrados a cada 5 min., desde t 0 a t 60 , para se avaliar a necessidade de doses anestésicas complementares. A FC foi aferida com estetoscópio e a FR através dos movimentos de expansão torácica.

Os animais que apresentaram apnéia por um período de tempo maior do que $60 \mathrm{seg}$. foram intubados e ventilados manualmente. Durante o período de apnéia não foram registrados os parâmetros clínicos.

Para as provas laboratoriais, as amostras de sangue foram divididas em três partes: um frasco contendo uma gota de EDTA $10 \%{ }^{3}$ para cada $3 \mathrm{ml}$ de sangue para realização do hemograma, para a confecção de dois esfregaços e do centrifugado para a obtenção de plasma e determinação de PPT e ALB; um frasco contendo uma gota de fluoreto de sódio $^{4}$ para cada $2,5 \mathrm{ml}$ de sangue para avaliação da glicemia e um frasco sem anticoagulante para obtenção de soro para determinação de ALT, AST, uréia e creatinina.

O hemograma foi realizado e as lâminas para leucometria específica foram coradas pelo método de Wright, segundo Jain (1986). O frasco com Glistab foi centrifugado ime-
1 LABTEST - MG, Brasil

2 Combur 8 Test - Boering Mannheim, Alemanha

3 Hemstab - LABTEST - MG, Brasil
4 Glistab - LABTEST - MG, Brasil

5 Espectrofotômetro SPEC 20D, Com \& Ind Dourado Ltda, SP, Brasil 
diatamente após a obtenção do sangue, a 3000 rpm por 5 min. e o plasma foi utilizado para determinação da glicemia. O frasco sem anticoagulante foi mantido inclinado por $2 \mathrm{~h}$, depois foi centrifugado e o soro obtido foi utilizado para a realização das análises bioquímicas, através de técnicas espectrofotométricas ${ }^{5}$ segundo Kaneko (1980).

Imediatamente após a coleta da urina, foram embebidas fitas regentes para a determinação simultânea de nitrito, $\mathrm{pH}$, proteína, bilirrubina, urobilinogênio, glicose, cetona e sangue, segundo Osborne et al. (1972). As leituras foram feitas após $60 \mathrm{seg}$.

Os dados obtidos receberam um tratamento estatístico de correlação linear e análise de variância; os resultados significativos a $1 \%$ foram comparados através do teste Duncan (programa SAEG 3.0).

\section{Resultados}

Os valores médios das doses de indução, manutenção e, conseqüentemente, a dose total obtidos com o uso do propofol foram 7,$3 ; 26,2$ e $33,5 \mathrm{mg} / \mathrm{kg}$, respectivamente. A duração da apnéia no teste de médias foi de 1,1 e 2 min., tendo ocorrido em dois animais $(28,6 \%)$ após a administração do propofol.

A FC/min. variou entre 80 e 160 e a FR entre 4 e 60 incursões/min.

Não foi possível a realização dos testes estatísticos de FC, FR e FP, em relação aos demais parâmetros aferidos, devido à falta de dados em alguns dos tempos estabelecidos no protocolo; a ocorrência de apnéia não permitiu a anotação desses valores. Foram realizados testes estatísticos entre t 0 e t 10 , não se encontrando diferenças significativas a 1\%, nos valores da FC e da FR após o uso do propofol.

Os valores de VG, concentração de hemoglobina globular média (CHGM) e $\mathrm{Hb}$ não tiveram diferenças significativas a $1 \%$ ao se compararem todos os parâmetros. De um modo geral, os valores médios dó VG e da $\mathrm{Hb}$ diminuíram enquanto que os da CHGM aumentaram após o propofol, porém todos oscilando dentro da faixa de normalidade (Tabela I).

Ao serem comparados todos os valores da hematimetria (Tabela I) não houve diferenças significativas a $1 \%$. Após o uso do propofol a hematimetria teve uma diminuição de seus valores médios. A leucometria global (LG) teve diferença significativa a $1 \%$ no teste de médias em relação aos tempos, sendo I, II; III diferentes de IV, e uma correlação positiva a $1 \%$ entre a contagem nos tempos I e II com $R=0,97$ (Tabela II).

Os valores das contagens absolutas dos leucócitos não apresentaram diferenças significativas a $1 \%$ ao se compararem todos os parâmetros.

Os valores médios dos basófilos diminuíram e os valores dos eosinófilos aumentaram após a administração do propofol. Os dos mielócitos permaneceram iguais a zero. Os dos basófilos e eosinófilos não apresentaram diferen-
Tabela 1 - Médias e desvios padrão do eritrograma nos 7 animais anestesiados com propofol nos diferentes tempos

\begin{tabular}{lcccc}
\hline & \multicolumn{5}{c}{ Propofol } \\
\hline $\begin{array}{l}\text { Tempos } \\
\text { Parâmetros }\end{array}$ & $\mathrm{I}$ & $\|$ & $\mathrm{II}$ & $\mathrm{N}$ \\
\hline Hemácias & & & & \\
(x 10\%/ml) & 4.8 & 3.5 & 5.4 & 4.7 \\
Volume globular & $(0.88)$ & $(0.47)$ & $(1.40)$ & $(0.94)$ \\
$\begin{array}{l}\text { (\%) } \\
\text { Hemoglobulinometria }\end{array}$ & 35.5 & 28.1 & 36.3 & 33.3 \\
(g/dl) & $(3.90)$ & $(3.60)$ & $(4.90)$ & $(5.60)$ \\
Vol. globular médio & $(1.80)$ & $(1.30)$ & $(1.50)$ & $(2.40)$ \\
(fl) & 74.1 & 80.0 & 70.3 & 72.0 \\
Concentração de & $(5.50)$ & $(7.50)$ & $(10.2)$ & $(7.90)$ \\
hemoglobina globular & 30.1 & 31.0 & 33.0 & 29.4 \\
média (\%) & & & & \\
\hline
\end{tabular}

Tabela 2 - Médias e desvios padrão do leucograma nos 7 animais anestesiados com propofol nos diferentes tempos

\begin{tabular}{lcccc} 
& \multicolumn{5}{c}{ Propofol } \\
\hline $\begin{array}{l}\text { Tempos } \\
\text { Parâmetros }\end{array}$ & $\mathrm{I}$ & $\mathrm{II}$ & $\mathrm{III}$ & $\mathrm{N}$ \\
\hline Leucometria global & 10.8 & 4.2 & 8.3 & 20.0 \\
(× 103/ml) & $(4.4)$ & $(5.1)$ & $(5.0)$ & $(9.3)$ \\
Bastões & 4.6 & 5.3 & 16.7 & 12.9 \\
$(\%)$ & $(4.9)$ & $(7.5)$ & $(7.4)$ & $(6.6)$ \\
Segmentados & 62.0 & 54.7 & 64.1 & 70.6 \\
$(\%)$ & $(7.0)$ & $(5.7)$ & $(5.6)$ & $(4.8)$ \\
Linfócitos & 14.7 & 17.7 & 7.4 & 4.9 \\
$(\%)$ & $(7.0)$ & $(7.9)$ & $(1.6)$ & $(2.6)$ \\
Monócitos & 5.9 & 3.9 & 3.0 & 7.3 \\
$(\%)$ & $(2.8)$ & $(2.0)$ & $(2.3)$ & $(3.5)$ \\
Eosinófilos & 12.3 & 17.9 & 7.1 & 4.1 \\
$(\%)$ & $(4.0)$ & $(9.0)$ & $(6.2)$ & $(3.8)$ \\
Basófilos & 0.6 & 0.6 & 0.6 & 0.3 \\
$(\%)$ & $(0.9)$ & $(0.9)$ & $(0.7)$ & $(0.7)$ \\
\hline
\end{tabular}

ças significativas a $1 \%$ ao se compararem todos os parâmetros (Tabela II).

Os valores médios dos linfócitos e dos monócitos diminuíram enquanto que os dos segmentados aumentaram. Não houve diferença significativa a $1 \%$ nos valores dos linfócitos, monócitos e segmentados, ao se compararem todos os parâmetros (Tabela II).

No teste de médias dos bastões em relação aos tempos houve diferença significativa a $1 \%$ sendo o no tempo 1 diferente daquele no tempo 1 II sob anestesia com propofol (Tabela II).

Os valores médios das PPT, das GLOB e ALB apre- 
Tabela 3 - Médias e desvios padrão da bioquímica sangüínea nos 7 animais anestesiados com propofol nos diferentes tempos

\begin{tabular}{|c|c|c|c|c|}
\hline \multirow[b]{2}{*}{$\begin{array}{l}\text { Tempos } \\
\text { Parâmetros }\end{array}$} & \multicolumn{4}{|c|}{ Propofol } \\
\hline & 1 & II & III & $N$ \\
\hline $\begin{array}{l}\text { Glicose } \\
\text { (mg/dl) }\end{array}$ & $\begin{array}{c}74.9 \\
(13.7)\end{array}$ & $\begin{array}{l}74.6 \\
(9.5)\end{array}$ & $\begin{array}{r}68.9 \\
(11.4)\end{array}$ & $\begin{array}{c}67.6 \\
(10.2)\end{array}$ \\
\hline $\begin{array}{l}\text { Proteínas plasm. totais } \\
(\mathrm{g} / \mathrm{dl})\end{array}$ & $\begin{array}{c}6.8 \\
(0.9)\end{array}$ & $\begin{array}{r}5.2 \\
(0.9)\end{array}$ & $\begin{array}{c}5.6 \\
(0.6)\end{array}$ & $\begin{array}{c}6.1 \\
(1.2)\end{array}$ \\
\hline $\begin{array}{l}\text { Globulinas } \\
(\mathrm{g} / \mathrm{dl})\end{array}$ & $\begin{array}{l}4.2 \\
(0.9)\end{array}$ & $\begin{array}{c}3.2 \\
(0.8)\end{array}$ & $\begin{array}{c}3.3 \\
(0.2)\end{array}$ & $\begin{array}{c}3.6 \\
(1.0)\end{array}$ \\
\hline $\begin{array}{l}\text { Albumina } \\
(\mathrm{g} / \mathrm{dl})\end{array}$ & $\begin{array}{c}2.6 \\
(0.3)\end{array}$ & $\begin{array}{c}2.0 \\
(0.3)\end{array}$ & $\begin{array}{c}2.3 \\
(0.5)\end{array}$ & $\begin{array}{r}2.6 \\
(0.8)\end{array}$ \\
\hline Albumina/globulina & $\begin{array}{c}0.6 \\
(0.2)\end{array}$ & $\begin{array}{c}0.7 \\
(0.2)\end{array}$ & $\begin{array}{c}0.7 \\
(0.1)\end{array}$ & $\begin{array}{c}0.8 \\
(0.4)\end{array}$ \\
\hline $\begin{array}{l}\text { Aspartato amino } \\
\text { transferase } \\
\text { (UI/I) }\end{array}$ & $\begin{array}{l}10.2 \\
(4.2)\end{array}$ & $\begin{array}{l}15.1 \\
(8.5)\end{array}$ & $\begin{array}{l}14.0 \\
(6.4)\end{array}$ & $\begin{array}{r}9.2 \\
(3.2)\end{array}$ \\
\hline $\begin{array}{l}\text { Alamina amino } \\
\text { transferase } \\
(\mathrm{Ul} / \mathrm{dl})\end{array}$ & $\begin{array}{l}10.5 \\
(4.5)\end{array}$ & $\begin{array}{l}18.4 \\
(9.8)\end{array}$ & $\begin{array}{c}18.7 \\
(12.3)\end{array}$ & $\begin{array}{c}19.8 \\
(13.8)\end{array}$ \\
\hline $\begin{array}{l}\text { Uréia } \\
(\mathrm{mg} / \mathrm{dl})\end{array}$ & $\begin{array}{l}16.1 \\
(7.7)\end{array}$ & $\begin{array}{c}23.9 \\
(12.6)\end{array}$ & $\begin{array}{c}23.3 \\
(16.5)\end{array}$ & $\begin{array}{l}24.9 \\
(8.8)\end{array}$ \\
\hline $\begin{array}{l}\text { Creatinina } \\
(\mathrm{mg} / \mathrm{dl})\end{array}$ & $\begin{array}{c}0.8 \\
(0.3)\end{array}$ & $\begin{array}{c}1.0 \\
(0.2)\end{array}$ & $\begin{array}{c}0.9 \\
(0.3)\end{array}$ & $\begin{array}{c}1.0 \\
(0.2)\end{array}$ \\
\hline
\end{tabular}

sentaram uma diminuição e, de modo inverso, o valor da ALB/GLOB teve aumento após o uso do propofol (Tabela III). Não houve diferença significativa a $1 \%$ destes valores e da GS ao se compararem todos os parâmetros.

Os valores da AST, da ALT e da creatinina não tiveram diferenças significativas a $1 \%$ ao se compararem todos os parâmetros (Tabela III). Por outro lado, foram encontrados valores médios aumentados da AST, da ALT, da uréia e da creatinina após o uso do propofol.

O nitrito, a proteína e o $\mathrm{pH}$ da urina não tiveram valores com diferenças significativas a $1 \%$ ao se compararem todos os parâmetros (Tabela IV). Os valores médios das proteínas apresentaram aumento, os da glicose permaneceram iguais a zero, os do nitrito negativaram-se e os do $\mathrm{pH}$ aumentaram após o uso do anestésico (Tabela IV). O valor do nitrito no animal 5 , igual a 1 , pode ter ocorrido em função de um erro na leitura da fita reagente.

Os valores médios da bilirrubina tiveram um discreto aumento enquanto que os do urobilinogênio e das cetonas foram iguais a zero. Os do sangue apresentaram aumento após administração do propofol (Tabela IV). Todos os valores dos parâmetros urinários não tiveram diferençaś significativas a $1 \%$ ao se compararem todos os parâmetros aferidos.
Tabela 4 - Médias e desvios padrão dos parâmetros urinários nos 7 animais anestesiados com Propofol nos diferentes tempos

\begin{tabular}{lcccc}
\hline & \multicolumn{4}{c}{ Propofol } \\
\hline $\begin{array}{l}\text { Tempos } \\
\text { Parâmetros }\end{array}$ & $\mathrm{I}$ & $\mathrm{II}$ & $\mathrm{Il}$ & $\mathrm{N}$ \\
\hline $\begin{array}{l}\text { Nitrito } \\
(\mathrm{g} / \mathrm{dl})\end{array}$ & 0.3 & 0.1 & 0 & 0 \\
Proteína & $(0.5)$ & $(0.3)$ & & \\
(g/dl) & 12.9 & 8.6 & 50.0 & 31.4 \\
$\mathrm{pH}$ & $(14.8)$ & $(13.6)$ & $(31.6)$ & $(30.9)$ \\
& 5.6 & 5.6 & 6.6 & 6.0 \\
Bilirrubina & $(1.0)$ & $(1.0)$ & $(1.2)$ & $(1.1)$ \\
& 0.6 & 0.6 & 0.9 & 0.6 \\
Sangue & $(0.5)$ & $(0.5)$ & $(0.8)$ & $(0.5)$ \\
(cel/ml) & 72.9 & 37.1 & 80.0 & 79.3 \\
& $(112.1)$ & $(86.9)$ & $(108.7)$ & $(109.2)$ \\
& & & &
\end{tabular}

Os valores do sangue podem ser em decorrência do cateterismo vesical, não indicando uma provável influência da droga sobre esse parâmetro urinário.

\section{Discussão}

A média das doses de indução com bolus, com propofol, sem pré-anestésico, foi de $7,3 \mathrm{mg} / \mathrm{kg}$, variando entre $6,7 \mathrm{e}$ $7,9 \mathrm{mg} / \mathrm{kg}$ neste trabalho e incluindo-se entre os limites de variação observados por outros autores (Watkins et al., 1987; Genevois et al., 1991) que foram de 4,09 a 7,81 mg/kg, 5,0 a $8,2 \mathrm{mg} / \mathrm{kg}, 6,98$ a $8,0 \mathrm{mg} / \mathrm{kg}$, e de 6,3 a $12,5 \mathrm{mg} / \mathrm{kg}$, respectivamente. Searle (1989) e Jones (1990) utilizaram $5,95 \mathrm{mg} / \mathrm{kg}$, enquanto Morgan e Legge (1989) empregaram uma dose próxima ao limite inferior deste trabalho: 6,55 $\mathrm{mg} / \mathrm{kg}$, e Mandsager et al. (1991) e Quandt e Robinson (1992) utilizaram $8,0 \mathrm{mg} / \mathrm{kg}$, próximos do limite superior adotado. Houve, portanto, uma semelhança entre as doses escolhidas para indução com bolus de propofol sem pré-anestésico, por aqueles autores, com as empregadas neste trabalho.

A técnica de manutenção anestésica, em bolus de propofol, apresentou resultado satisfatório na dose de 26,2 $\mathrm{mg} / \mathrm{kg}$, correspondendo a $0,437 \mathrm{mg} / \mathrm{kg} / \mathrm{min}$. em $60 \mathrm{~min}$., estando entre os limites de variação da dose de manutenção observada por Watkins et al. (1987): de 0,393 a 1,219 $\mathrm{mg} / \mathrm{kg} / \mathrm{min}$. e sendo menor do que as indicadas por outros autores (Morgan e Legge, 1989; Searle, 1989; Jones, 1990; Quandt e Robinson, 1991), que foram de $1,296 \mathrm{mg} / \mathrm{kg} / \mathrm{min}$., $0,81 \mathrm{mg} / \mathrm{kg} / \mathrm{min}$., 0,806 mg/kg/min., e 0,6 mg/kg/min. (nos primeiros $30 \mathrm{~min}$.) e $0,8 \mathrm{mg} / \mathrm{kg} / \mathrm{min}$. (dos 31 aos $60 \mathrm{~min}$.), respectivamente.

A apnéia ocorreu em 2 dos 7 animais (28\%); Watkins et al. (1987) observaram uma ocorrência virtualmente me- 
nor (11 de 68 animais - 16,2\%) enquanto Quandt e Robinson (1991) e Mandsager et al. (1991) observaram uma ocorrência virtualmente maior do que a enccritrada nesse trabalho (2 de $5-40 \%$ e 5 de 10 animais - 50\%, respectivamente). $O$ tempo de duração da apnéia nos animais sob efeito do propofol foi de 2 e 1,1 min., com valores semelhantes aos encontrados por Marsico et al. (1991), de 60 seg. a $3 \mathrm{~min}$. e maiores do que os observados por Watkins et al. (1987), de 30 seg. e Genevois et al. (1988), de 10 a 30 seg.

Os resultados obtidos na presente pesquisa caracterizam-se por um aumento da FC após a indução com bolus de propofol, estando de acordo com os encontrados por Marsico et al. (1991), Quandt e Robinson (1992), e diferentes dos de Mandsager et al. (1991), que observaram bradicardia sinusal como efeito do propofol. Por outro lado, Quandt e Robinson (1992) não encontraram alterações significativas nos parâmetros cardiovasculares após anestesia com propofol, assemelhando-se aos nossos resultados. A FR não teve diferenças significativas a $1 \%$ após indução anestésica; no entanto, constatou-se uma depressão respiratória após a utilização do propofol, confirmando os resultados obtidos por Marsico et al. (1991) e Quandt e Robinson (1991).

O período anestésico adotado, 60 min., está de acordo com Mandsager et al. (1991) e Quandt e Robinson (1992). Já o intervalo de $10 \mathrm{~min}$. entre as aferições de FC e FR, divergiu do de Mandsager et al. (1991) que aferiram de 5 em 5 min. e do de Marsico et al. (1991) que, ao trabalharem apenas com indução anestésica, aferiram também a cada 5 min. entre os tempos 0 e $15 \mathrm{~min}$.

As coletas de sangue e urina foram realizadas no tempo I (permitindo a obtenção de parâmetros laboratoriais sem efeito do anestésico), no tempo II (ao término da anestesia), no tempo III (quando há a segunda fase de eliminação do propofol no homem) e no tempo IV (24 h após a indução anestésica).

Segundo estudo hematológico em cinco ensaios (Wellcome, 1989) nenhum paciente humano submetido a cirurgia sob anestesia com propofol teve os valores de uréia ou creatinina aumentados acima da faixa de normalidade, respectivamente em 162 e 158 pacientes avaliados, não havendo evidências claras de que o propofol afete a função renal. Neste trabalho observou-se que, após o uso do propofol, 2 animais apresentaram em relação ao tempo I aumento dos valores de uréia acima da faixa de normalidade que não foram significativos a $1 \%$ ao se compararem todos os parâmetros e assemelharam-se às observações do Laboratório Wellcome (1989). Do mesmo modo, não se encontraram valores de creatinina fora da faixa de normalidade.

Houve uma correlação negativa a $1 \%$ entre os valores do volume globular médio (VGM) e a dose de indução com propofol no tempo III. O animal 3 teve uma diminuição do VGM, abaixo da faixa de normalidade, no tempo III (53) em relação aos tempos I (81) e II (85), tendendo a um valor normal no tempo IV (60), que pode ser devido à hemoconcentração no tempo III, pois os valores das hemácias, do VG, da uréia e das PPT apresentaram um aumento no tempo III em relação aos tempos I e II. Como foram verificadas alterações numéricas consideráveis nos valores das hemácias e do VG, podem ter ocorrido variações fisiológicas com influência direta sobre o valor do VGM. Como o propofol, provavelmente, foi eliminado entre 25 e $56 \mathrm{~min}$. e posteriormente entre 184 e $309 \mathrm{~min}$., em eliminação bifásica, como ocorre no homem (Langley e Heel, 1988), não se justifica haver alterações nos valores do VGM no tempo III por influência do anestésico.

Com a utilização do propofol, os valores da LG apresentaram uma correlação positiva a $1 \%$ entre os tempos I $\left(10 \times 10^{3} \pm 4,4 \times 10^{3}\right)$ e II $\left(4,2 \times 10^{3} \pm 5,1 \times 10^{3}\right)$. No tempo II, 1 animal apresentou a LG com valor normal, enquanto que os 6 restantes tiveram leucopenia; desses 2 tiveram desvio à esquerda sem neutrofilia, 1 apresentou valores normais de neutrófilos e 3 tiveram valores de segmentados abaixo da faixa de normalidade. Houve diminuição, em todos os animais, dos valores da $L G$ no tempo II em relação ao tempo I; no tempo III $\left(8,3 \times 10^{3} \pm 5,0 \times 10^{3}\right) 6$ apresentaram diminuição dos valores em relação ao tempo I, sendo 3 leucopênicos; no tempo IV $\left(20 \times 10^{3} \pm 9,3, \times 10^{3}\right) 4$ animais tiveram aumento dos valores da $L G$ em relação ao tempo I, 1 teve diminuição e 1 permaneceu com valor igual; 4 animais evidenciaram leucocitose sem alteração de segmentados e 1 teve leucocitose neutrofílica. De um modo geral, observou-se diminuição dos valores da $L G$ nos tempos II $\left(4,2 \times 10^{3} \pm 5,1 \times 10^{3}\right)$ e III $\left(8,3 \times 10^{3} \pm 5,0 \times 10^{3}\right)$ e aumento no tempo IV $\left(20 \times 10^{3} \pm 9,3 \times 10^{3}\right)$ em relação ao tempo I $\left(10 \times 10^{3} \pm 4,4 \times 10^{3}\right)$.

Coles (1984) enumera prováveis causas da leucopenia, acreditando entre outras, estar associada com estado de debilidade e deficiência nutricional, porém, neste trabalho, 5 animais não se encontravam leucopênicos no tempo I.

Segundo estudo hematológico em cinco ensaios (Wellcome, 1989), de 157 pacientes humanos submetidos à cirurgia sob anestesia com propofol 2 tiveram os valores da $L G$ abaixo da faixa de normalidade, mas já estavam com valores baixos antes da anestesia; concluíram também, que não existem evidências claras de que o propofol afete os parâmetros hematológicos.

Sob anestesia com propofol, a LG apresentou uma diferença significativa a $1 \%$ no teste de médias, em relação aos tempos, quando I, II e III foram diferentes de IV. A diminuição significativa nos tempos II e III, estando de acordo com os dados do laboratório Wellcome (1989), provavelmente, ocorreu devido à liberação de substâncias vasoativas, e não por efeito direto da droga, ou seja, por causa do seqüestro de células no pool marginal e/ou devido à diapedese facilitada pela droga. $\mathrm{O}$ aumento no tempo IV, provavelmente ocorreu por inibição da marginalização, determinando a liberação das células já 
existentes no tempo I no pool circulante, acrescidas das células produzidas na medula óssea nesse período, que também ficaram seqüestradas no pool marginal.

No mesmo estudo hematológico realizado pelo Laboratório Wellcome (1989), em 149 pacientes humanos 5, que já se encontravam anêmicos antes da anestesia, tiveram valores abaixo da faixa de normalidade.

Em todos os animais submetidos à anestesia com propofol, foram encontrados valores do $V G$ abaixo da faixa de normalidade; 4 tiveram valores de VG $(35,4 \pm 3,9)$ e hemácias $\left(4,80 \times 10^{6} \pm 0,88 \times 10^{6}\right)$ abaixo da faixa de normalidade no tempo $\mathrm{I}$, indicando que encontravam-se anêmicos no período pré-anestésico. Como em todos os animais da presente pesquisa houve uma diminuição dos valores do VG no tempo II $(28,1 \pm 3,6)$ em relação ao tempo I, ficando todos abaixo da faixa de normalidade, acredita-se que a diminuição ocorreu devido a alterações hemodinâmicas e não por efeito do anestésico, já que no tempo III $(36,3 \pm 4,9), 4$ animais tiveram os valores aumentados em relação ao tempo $\mathrm{I}$.

O Laboratório Wellcome (1989) observou, em estudos realizados em pacientes humanos, que o propofol apresentou um alto grau de ligação às proteínas plasmáticas. Segundo Hall e Clarke (1987), pode haver diminuição das PPT em alguns casos de anemia. Em nosso trabalho, observamos que 4 animais tinham anemia no tempo I, enquanto que somente 1 destes tinha valor de PPT abaixo da faixa de normalidade, e uma dose total menor do que a dose média de cães anestesiados com propofol, aparentemente respondendo mais à droga.

Todos os animais anestesiados com propofol tiveram valores de AST (10,2 $\pm 4,2)$ e de $\operatorname{ALT}(10,0 \pm 7,7)$ abaixo da faixa de normalidade no tempo $\mathrm{I}$, mas aumentados nos tempos II $(15,1 \pm 8,5$ e $8,3 \pm 4,3)$, III $(14,0 \pm 6,4$ e $9,1 \pm$ $4,0)$ e IV $(9,2 \pm 3,2$ e $9,0 \pm 2,9)$, isto é, permaneceram abaixo ou ficaram dentro da faixa de normalidade. Estes resultados estão de acordo com os encontrados pelo Laboratório Wellcome (1989), quando nenhum paciente humano teve valores acima da faixa de normalidade, e pontificam que não há evidências de que o propofol afete adversamente a função hepática.

\section{Conclusões}

Pelo presente estudo podemos concluir que o propofol não determinou alterações significativas hepáticas, urinárias, renais e no eritrograma; que determinou desvio nuclear de neutrófilos à esquerda sem neutrofilia nos quatro tempos, havendo uma importante influência sobre este parâmetro; que houve diminuição dos valores das proteínas plasmáticas totais, globulinas e albuminas, com provável comprometimento no transporte do propofol no sangue.

\section{Abstract}

\section{Propofol: clinical and laboratorial evaluation in dogs}

Seven dogs (Canis familiaris) were induced and maintained in anesthesia bolus of propofol and thiopental, individually, without pre-anesthesia for $60 \mathrm{~min}$. It were evaluated clinical parameters: cardiac, breath, and pulse frequence/min., eye-lid and inter-finger reflexes; laboratorial parameters: hematological - erytrocytes, paeked cell volume, haemoglobin concentration, mean corpuscular volume, mean corpuscular haemoglobin concentration, total leucocyte counts, and specific leucocyte counts; biochemical - aspartate aminotranferase, alanine aminotransferase, urea, creatinine, glucose, total plasmatic protein, albumin, globulins, and albumin/globulin ratio; and urinary - nitrite, $\mathrm{pH}$, protein, glucose, cetones, urobilinogen, bilirrubin, and blood by data colected on pré-established times, behind the anesthesia induction, to $24 \mathrm{hr}$ after it.

It was concluded that the propofol caused significative alterations in values of total leucocyte counts, in slapstick neutrophyl, and in pulse frequence.

Key words: propofol; thiopental; anesthesia; hemathology; biochemistry; urine; dog

\section{Referências bibliográficas}

COLES, E. H. Patologia Clínica Veterinária, $3^{a}$ ed., São Paulo, Manole, 1984, $566 \mathrm{p}$.

GENEVOIS, J. P., FAU, D., FIENI, F.,TAINTURIER, D., HOSSEINZADEH, G., GUYONNET, V. Use of a new anaesthetic in dog: P. 1. Results after the injection of a single dose. Rev. Méd. Vét., v. 139, n. 12, p. 1119-1123, 1988.

HALL, L. W., CLARKE, K. W. Anestesia Veterinária, $8^{\mathrm{a}}$ ed. São Paulo, Manole, 1987, $415 \mathrm{p}$.

JAIN, N. C. Schalm's Veterinary Hematology. 4. ed, Lea \& Febiges, Philadelphia, 1986, $1221 \mathrm{p}$.

JONES, R. S. Propofol: anaesthesia in the dog and cat. Veterinary Annual, Liverpool Royal Infirmary, v. 30, p. 200202, 1990.

KANECO, J. J. Clinical Biochemestry of Domestic Animals. 3. ed., Academic Press, New York, 1980, 831 p.

LANGLEY, M. S., HEEL, R. C. Propofol: a review of its pharmacodynamic and pharmacokinetic properties and use as an intravenous anaesthetic. Drugs, v. 35 , p. 334372, 1988.

MANDSAGER, R. E., SHAWLEY, R. V., CLARK, D. M. Evaluation of Propofol or acepromazine/diazepan/etamine in the Greyhound. Vet. Surgery, v. 20, p. 80-81, 1991.

MARSICO, F., TENDILLO, F. J., ALVAREZ, I. Un nuevo anestésico intra-venoso: Propofol. Evaluacion clinica y experimental. Clín. Vet. Peq. Animales, v. 11, n. 4, p. 231-239, 1991.

MORGAN, D. W. T., LEGGE, K. Clinical evaluation of Propofol as an intravenous anaesthetic agent in cats and dogs. Vet. Record, v. 124, n. 2, p. 31-33, 1989.

OSBORNE, C. A., LOW, D. G., FINCO, D. R. Canine and Feline Urology. W. B. Saunders, Philadelphia, 1972, 417 p. 
QUANDT, J. E., ROBINSON, E. P. Propofol: constant infusion anesthesia in dogs. Vet. Surgery, v. 20, n. 1, p. 81, 1991.

QUANDT, J. E., ROBINSON, E. P. Propofol and thiopental: comparison of anesthetic effects in the dog. Vet. Surgery, $v$. 21, n. 1, p. 83-84, 1992.

ROBERTSON, S. A., JOHNSTON, S., BEEMSTERBOER, J. A comparison of Propofol infusion anesthesia in Greyhound dogs. Vet. Surgery, v. 20, n. 2, p. 159, 1991.
SEARLE, A. P. Australian Veterinary Practioner, v. 19, n. 4, p. 216, 1989.

WATKINS, S. B., HALL, L. W., CLARKE, K. W. Propofol as an intravenous anaesthetic agent in dogs. Vet. Record, v. 120, n. 14, p. 326-329, 1987.

WELLCOME. Diprivan, Propofol. O anestésico venoso versátil. Laboratórios Wellcome - ICI Ltda., Cotia, São Paulo, Brasil, 1989. 